\title{
HIGH SENSITIVITY C-REACTIVE PROTEIN IN ACUTE ISCHAEMIC STROKE
}

\author{
Gurunamasivayam Gurusamy1, Sivaraman $R^{2}$
}

${ }^{1}$ Senior Assistant Professor, Department of Internal Medicine, Government Kilpauk Medical College \& Hospital, Chennai. ¿Senior Resident, Department of Internal Medicine, Government of Kilpauk Medical College \& Hospital, Chennai.

\section{ABSTRACT}

\section{BACKGROUND}

Elevated levels of hs-CRP are found to be related with higher risk of first ever cardiovascular, cerebrovascular and peripheral vascular diseases and also initial CRP levels carry prognostic value in acute ischaemic stroke \& acute coronary syndrome. This study aims to describe such relation.

\section{MATERIALS AND METHODS}

In 50 acute ischaemic stroke patients, initial 48 hours hsCRP levels were measured and patients were followed up after 4 weeks to see their neurological status by Glasgow Outcome Scale. Also, we studied the levels of hs-CRP in relation to various risk factors of stroke.

\section{RESULTS}

Out of the $34 \%$ cases with favourable outcome, $30 \%$ had CRP $<10.1 \mathrm{mg} / \mathrm{L}$ and $4 \%$ had CRP $>10.1 \mathrm{mg} / \mathrm{L}$. All the remaining $66 \%$ cases with unfavourable outcome had hs-CRP $>10.1 \mathrm{mg} / \mathrm{L} . \mathrm{P}=0.000$.

\section{CONCLUSION}

Initial levels >10.1 predicts poor outcome in stroke; hs-CRP levels showed statistically significant elevation in patients with hypertension; hs-CRP levels had no significant correlation with age, gender, smoking, cholesterol intake, diabetes or IHD.

\section{KEYWORDS}

Stroke, hs-CRP, Glasgow Outcome Scale, Neurological Outcome.

HOW TO CITE THIS ARTICLE: Gurusamy G, Sivaraman R. High sensitivity C-reactive protein in acute ischaemic stroke. J. Evolution Med. Dent. Sci. 2017;6(5):426-429, DOI: 10.14260/Jemds/2017/94

\section{BACKGROUND}

In recent years, there has been increasing evidence which shows strong links between inflammation and the pathogenesis of atherothrombotic stroke. Acute phase proteins have been implicated to play roles both during acute and chronic inflammatory processes in different diseases including ischaemic stroke.

There is growing evidence of the prognostic importance of CRP in ischaemic stroke.(1) Also CRP has been found to be a strong but relatively non-specific risk factor of fatal stroke in elderly persons.

CRP, a sensitive meter of inflammation, induces vascular thrombosis by stimulating monocytes ${ }^{(2)}$ to express tissue factor, the initiator of the extrinsic pathway of coagulation. Elevated levels of CRP are found to be related with higher risk of first ever cardiovascular, cerebrovascular and peripheral vascular diseases.

Financial or Other, Competing Interest: None.

Submission 11-12-2016, Peer Review 04-01-2017,

Acceptance 10-01-2017, Published 16-01-2017.

Corresponding Author:

Dr. Gurunamasivayam Gurusamy,

A.1\#8, "Coconut Grove Residency"

12th Street, Tansi Nagar,

(Near Behind Church),

Velachery,

Chennai-600042.

E-mail: sudhaguru1@gmail.com

DOI: $10.14260 /$ jemds $/ 2017 / 94$

\section{(c) $(1)(-$}

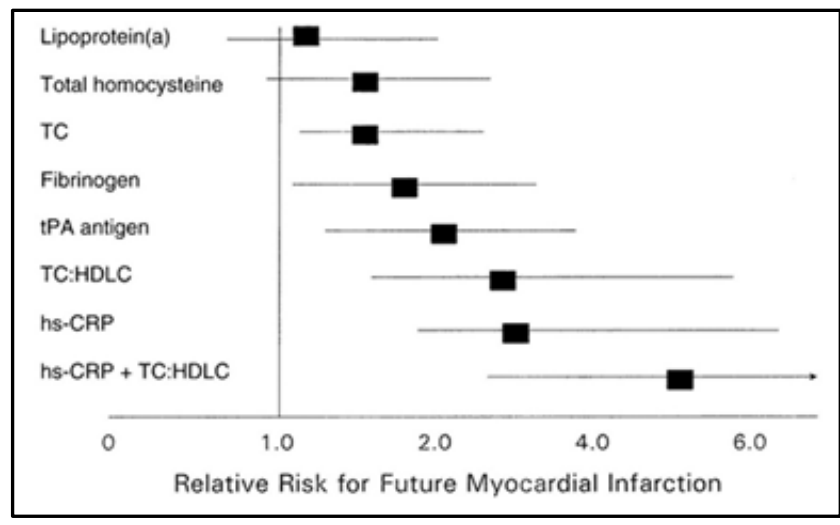

Figure 1. Prognostic Value of Acute Phase Reactants (APR)

CRP has been used to understand the inflammatory components of atherosclerosis than other inflammatory mediators such as IL-6 \& TNF -alpha.

CRP levels rapidly increase after inflammatory stimulus and depending upon the intensity of the stimulus, even a 100 fold increase in plasma levels may occur. CRP is not degraded to a significant extent in any process and its clearance is not influenced by any condition. Hence, its concentration appears to be dependent on the rate of production and elimination. The $\mathrm{t}^{1 / 2}$ is 19 hours, which is long and makes its detection in blood after several hours of stimulus. Because of this nature of CRP, it is called as an "ideal marker of inflammation".

Acute phase proteins include, CRP, albumin, alpha 1 antitrypsin, pro-calcitonin, ceruloplasmin, alpha 1 acid 
glycoprotein, fibrinogen, haptoglobin, alpha-2 haptoglobin, and serum amyloid A.

The disadvantage of the above-mentioned proteins is that the difference in normal and pathological values are negligible.

High sensitivity CRP assays measure serum concentrations at the lower end.

\begin{tabular}{|c|c|c|c|c|}
\hline & Unit & Normal & Moderate & High Risk \\
\hline \multirow{2}{*}{ Hs-CRP } & $\mathrm{mg} / \mathrm{dl}$ & $<1$ & $1-3$ & $>3$ \\
\cline { 2 - 5 } & $\mathrm{mg} / \mathrm{L}$ & $<10$ & $10-30$ & $>30$ \\
\hline
\end{tabular}

\section{Table 1. Hs-CRP in Risk Assessment}

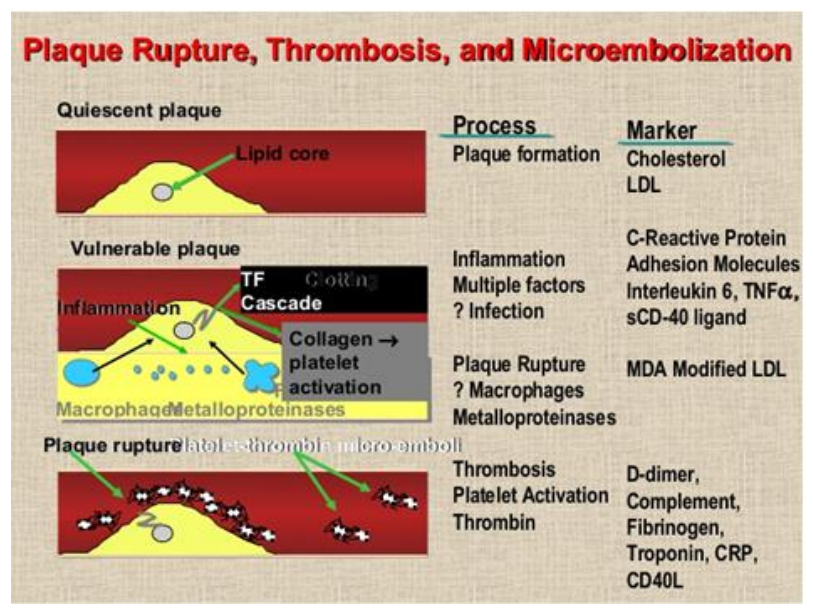

Figure 2. Vascular Pathology \& Utility of markers

\section{This Study Aims}

1. To evaluate the predictive value of hs-CRP in relation to first ever ischaemic stroke.

2. To correlate the hs-CRP levels with various cardiovascular risk factors.

\section{MATERIALS AND METHODS}

\section{Inclusion Criteria}

All patients who presented within 48 hours of onset of stroke.

\section{Exclusion Criteria}

1. Haemorrhagic stroke.

2. Age $>70$.

3. Evidence of active infection and neoplastic conditions.

4. Rheumatic heart disease and collagen vascular disease.

5. Patients who were actively smoking at the time of study.

6. Patients with previous history of TIA.

\section{Study Method}

A total of 50 patients who presented with acute ischaemic stroke were enrolled into the study. That the stroke was an ischaemic one was confirmed by CT scan. As soon as the patients were admitted within 48 hours of onset of stroke, serum samples were taken for hs-CRP estimation. Standard guidelines for the treatment of acute ischaemic stroke were followed. None of the patients received any thrombolytic treatment. They were treated only with anti-oedema measures and antiplatelet drugs such as aspirin alone and with good nursing care and physiotherapy. The patients were reviewed after 4 weeks after onset of stroke and were stratified using the Glasgow Outcome Scale (GOS). The serum
hs-CRP level was correlated with the functional recovery of patients after 4 weeks using the GOS.

The GOS was utilised to assess the functional outcome and residual neurological deficit. It has frequently been used in trials involving stroke and brain injuries. It is a wellvalidated scale with good interobserver agreement.

\section{Glasgow Outcome Scale (GOS)-}

1. Death.

2. A vegetative state.

3. The patient is unable to live independently, but can follow commands.

4. The patient is capable of living independently, but unable to return to work or school.

5. The patient is able to return to work or school.

- Score of 4 and $5=$ Good outcome.

- Score of 1,2, 3 = Poor outcome.

Cut- off value for hs-CRP for assessing the prognosis of stroke in this study was taken as $\geq 10.1 \mathrm{mg} / \mathrm{L}$ and the serum hs-CRP level was correlated with the functional recovery of patients after 4 weeks using the GOS. This was based on a study by Muir KW et al who found that hs-CRP levels above $10.1 \mathrm{mg} / \mathrm{L}$ when measured within 72 hours of stroke predicted mortality over 4 years.

Hypertension in this study was taken as BP $\geq 140 / 90$ mmHg as per JNC VIII.

\section{Measurement of hs-CRP}

The Quantia CRP-US (from Tulip Diagnostics Pvt. Ltd) was used for the measurement of hs-CRP. Quantia CRP is a turbidimetric immunoassay for ultrasensitive determination of CRP in human serum and is based on the principle of agglutination reaction. The test specimen is mixed with Quantia CRP US latex reagent and activation buffer and allowed to react. Presence of CRP in the test specimen results in the formation of an insoluble complex producing a turbidity, which is measured at wavelength between 505-578 $\mathrm{nm}$. The increase in turbidity corresponds to the concentration of CRP in the test specimen.

The Quantia CRP US reagent has been designed to measure CRP concentrations in the range of $0.015-1.0 \mathrm{mg} / \mathrm{dL}$ and is linear within the measuring range.

\section{Statistical Analysis}

The collected data was analysed with SPSS 16.0 version. To describe about the data descriptive statistics, frequency analysis, percentage analysis were used for categorical variables and the mean \& S.D. were used for continuous variables. To find the significant difference between the bivariate samples in independent groups (Male \& Female) unpaired sample t-test was used. To find the significance in categorical data Chi-Square test was used. In both the above statistical tools, the probability value 0.05 is considered as significant level.

RESULTS
\begin{tabular}{|c|c|c|c|}
\hline GOS & \multicolumn{1}{|c|}{ hs-CRP (mg/L) } & Total \\
\hline & $<\mathbf{1 0 . 1}$ & $>\mathbf{1 0 . 1}$ & \\
\hline 1 & $0(0 \%)$ & $2(4 \%)$ & $2(4 \%)$ \\
\hline 2 & $0(0 \%)$ & $13(26 \%)$ & $13(26 \%)$ \\
\hline 3 & $0(0 \%)$ & $17(34 \%)$ & $17(34 \%)$ \\
\hline 4 & $7(14 \%)$ & $3(6 \%)$ & $10(20 \%)$ \\
\hline 5 & $8(16 \%)$ & $0(0 \%)$ & $8(16 \%)$ \\
\hline Total & $\mathbf{1 5 ( 3 0 \% )}$ & $\mathbf{3 5}(\mathbf{7 0} \%)$ & $\mathbf{5 0}(\mathbf{1 0 0} \%)$ \\
\hline \multicolumn{5}{|c|}{ Table 2. hs-CRP Vs GOS Score } \\
\hline
\end{tabular}


Out of the $34 \%$ cases with GOS score of 4 or 5 , i.e. those with favourable outcome, $30 \%$ had CRP $<10.1 \mathrm{mg} / \mathrm{L}$ and $4 \%$ had CRP > $10.1 \mathrm{mg} / \mathrm{L}$.

All the remaining $66 \%$ cases with GOS score of 1,2 or 3 , (i.e. unfavourable outcome) had hs-CRP $>10.1 \mathrm{mg} / \mathrm{L}$. $\mathrm{P}=0.000$.

The correlation between hs-CRP levels measured within 48 hours of onset of stroke to that of the functional outcome of the patient at the end of 4 weeks (using GOS) was carried out.

Out of the 50 cases enrolled in the study, 35 cases (70\%) had CRP values > $10.1 \mathrm{mg} / \mathrm{L}$ and 15 cases (30\%) had CRP < $10.1 \mathrm{mg} / \mathrm{L}$.

Out of the 35 cases with CRP > $10.1 \mathrm{mg} / \mathrm{L}, 4 \%$ had GOS score of $1,26 \%$ cases had GOS score of $2,34 \%$ cases had GOS score of 3.

Of the remaining 15 cases with CRP $<10.1 \mathrm{mg} / \mathrm{L}$, none had a GOS score of 1,2 or $3.14 \%$ cases had a GOS score of 4 and $16 \%$ cases had a GOS score of 5 .

Out of the 50 patients, 2 died. Both of them had very high hs-CRP levels.

\section{In our study, following Data was Observed}

1. Mean age of the patients included in this study was $\mathbf{5 9 . 9 0}$ with SD of 7.360 .

2. Mean age of female patients was 60.65 with SD 6.852 .

3. Mean age for male patients was 59.08 with Std. deviation of 7.940 .

4. Out of 26 females enrolled in this study, 15 had high HsCRP level and remaining 9 had normal hs-CRP level.

5. Out of 24 males enrolled in this study, 19 had high levels and remaining 5 had normal levels.

6. Mean time of sample collection is $\mathbf{1 3 . 9 6}$ with SD of 9.95 hrs.

7. In this study out of 34 patients, 31 people were in unfavourable group.

$P$ value - highly significant $=0.000$.

8. Out of 29 non-smokers, 17 had high value and 12 had normal value and out of 21 smokers, 17 had high value and 4 had normal value.

$P$ value- 0.095 , non-significant.

9. Out of 29 smokers 13 had favourable GOS score, remaining 16 had unfavourable score and out of 21 smokers, 5 had favourable score and remaining 16 had unfavourable score.

$P$ value -0.126 , not significant.

10. Out of 31 non-alcoholics in this study 19 had high levels of hs-CRP, remaining 12 had normal levels and out of 19 alcoholics, 15 had high values and remaining 4 had normal value.

$P$ value- 0.194 , not significant.

11. Out of 31 non alcoholics, 14 had favourable GOS score, remaining 17 had unfavourable score and out of 19 alcoholics, 4 had favourable GOS score and remaining 15 had unfavourable score.

$P$ value -0.089 , non-significant.

12. Out of 30 persons with high BP 24 had high hs-CRP levels and 6 had normal levels .out of 20 with normal BP 10 had high levels and the remaining 10 had normal levels

$P$ value -0.026 , significant.

13. Out of 30 hypertensives, 8 had favourable GOS score and 22 had unfavourable score. Out of 20 non-hypertensives,
10 had favourable score and remaining 10 had unfavourable score.

$P$ value- 0.092 , not significant.

14. Out of 15 patients, 14 had high levels of hs-CRP, remaining one patient had normal level. Out of 35 patients with normal serum cholesterol levels, 20 had high hs-CRP values, remaining 15 had normal values.

$P$ value- 0.012 , highly significant.

15. Out of 35 patients who had normal serum cholesterol level, 16 patients had favourable score and remaining 19 had unfavourable score. Out of 15 patients who had high serum cholesterol level, 2 had favourable score and 13 had unfavourable score.

$P$ value- 0.029 , significant.

16. Out of 40 patients who had normal cardiac status, 26 had high hs-CRP levels and 14 had normal level. Out of 10 cardiac patients, 8 had high levels and 2 had normal levels.

$P$ value -0.363 , not significant.

17. Out of 40 non-cardiac patients, 16 had favourable GOS score and 24 had unfavourable score. Out of 10 cardiac patients, 2 had favourable score and 8 had unfavourable score.

P value- 0.239 , not significant.

This is consistent with the various studies conducted using hs-CRP as a prognostic indicator of acute ischaemic stroke.

\section{DISCUSSION}

There was no statistically significant correlation between age and gender of the patient with the hs-CRP levels. This may be because of the small sample size of our study group. But in a study conducted by So Yeon Ryu et al(3) in 2005, age had an independent association with plasma hs-CRP whereas gender showed no significant association with plasma hs-CRP.

In our study, smoking and alcohol had no statistical significance with hs-CRP levels. This is in contrast to a study conducted by So Yeon Ryu et al(3)in 2005, where smoking had a significant correlation with plasma hs-CRP levels and moderate alcohol consumption reduces circulating hs-CRP.

In our study, total serum cholesterol did not have statistically significant correlation with hs-CRP levels. This is in contrast to a study by So Yeon Ryu et al.(3) A limitation of our study was that only total serum cholesterol was estimated and not the complete lipid profile.

Our study showed a statistically significant correlation between high BP and hs-CRP. This is consistent with the study by So Yeon Ryu et al (3) and Blackburn R et al.(4)

Diabetes had no statistical significance with hs-CRP in our study in contrast to many of the previous studies. This may be because of the small sample size and also because of the fact that our study included only acute ischaemic stroke patients; it was not done exclusively on diabetic subjects.

Our study also did not show a statistically significant correlation between IHD and hs-CRP levels. This is in contrast to many of the previous studies. $(5,6,7,8)$. This may be because our study included only ischaemic stroke patients, it did not include IHD cases without stroke.

The major difference between our study and those of other studies mentioned above was that our study involved only hs-CRP levels and not other acute phase reactants like 
fibrinogens. Also, in our study we assessed the outcome of patients with acute ischaemic stroke at the end of four weeks and not at the end of one year. Also, we measured the CRP levels only within 48 hours after the onset of ischaemic stroke and not at the end of four weeks or at the time of discharge. This was because of the cost involved in the measurement of hs-CRP. The other reason, why our study did not have significant correlation with age, sex, DM, IHD was that our study sample size was very small.

Knowledge of the prognostic influence of the levels of CRP in the outcome of stroke of atherothrombotic aetiology helps the clinician to offer realistic expectations to the families of stroke victims. (9)

As CRP was found to be an independent risk indicator of further cardiovascular and neurovascular events, routine CRP screening of susceptible population like chronic smokers and siblings and first degree relatives of patients with IHD and stroke may prove a valuable indicator for predicting future atherothrombotic events and then it can be assessed as a routine indicator for aspirin prophylaxis. (10)

\section{CONCLUSION}

- hs-CRP levels showed statistically significant elevation in patients with high blood pressure.

- hs-CRP levels had no significant correlation with age or gender.

- hs-CRP did not show a statistically significant correlation with smoking or cholesterol intake.

- There was no statistically significant correlation between hs-CRP levels and those with diabetes or IHD.

\section{REFERENCES}

[1] Cao JJ, Thach C, Manolio TA, et al. C-reactive protein, carotid intima media thickness, and incidence of ischemic stroke in the elderly: the cardiovascular health study. Circulation 2003;108(2):166-70.
[2] Cermak J, Key NS, Bach RR, et al. C-reactive protein induces human peripheral blood monocytes to synthesize tissue factor. Blood 1993;82(2):513-20.

[3] Ryu SY, Lee YS, Park J, et al. Relations of plasma highsensitivity C-reactive protein to various cardiovascular risk factors. J Korean Med Sci 2005;20(3):379-83.

[4] Blackburn R, Giral P, Bruckert E, et al. Elevated Creactive protein constitutes an independent predictor of advanced carotid plaques in dyslipidemic subjects. Arteriosclerosis Thrombosis and Vascular Biology 2001;21(12):1962-8.

[5] Biasucci LM, Liuzzo G, Grillo RL, et al. Elevated levels of C-reactive protein at discharge in patients with unstable angina predict recurrent instability. Circulation 1999;99(7):855-60.

[6] Choi H, Cho DH, Shin HH, et al. Association of high sensitivity C-reactive protein with coronary heart disease prediction, but not with carotid atherosclerosis, in patients with hypertension. Circ J 2004;68(4):297303.

[7] Rifai N, Ridker PM. High-sensitivity C-reactive protein: a novel and promising marker of coronary heart disease. Clin Chem 2001;47(3):403-11.

[8] Ridker PM, Buring JE, Shih J, et al. Prospective study of C-reactive protein and the risk of future cardiovascular events among apparently healthy women. Circulation 1998;98(8):731-3.

[9] Castillo J, Davalos A, Marrugat J, et al. Timing for feverrelated brain damage in acute ischemic stroke. Stroke 1998;29(12):2455-60.

[10] Magyar MT, Szikszai Z, Balla J, et al. Early-onset carotid atherosclerosis is associated with increased intimamedia thickness and elevated serum levels of inflammatory markers. Stroke 2003;34(1):58-63. 